\title{
The effect of the equivalent-weights particle filter on dynamical balance in a primitive equation model
}

Article

Published Version

Ades, M. and Van Leeuwen, P. (2015) The effect of the equivalent-weights particle filter on dynamical balance in a primitive equation model. Monthly Weather Review, 143 (2). pp. 581-596. ISSN 0027-0644 doi:

https://doi.org/10.1175/MWR-D-14-00050.1 Available at https://centaur.reading.ac.uk/40382/

It is advisable to refer to the publisher's version if you intend to cite from the work. See Guidance on citing.

Published version at: http://dx.doi.org/10.1175/MWR-D-14-00050.1

To link to this article DOI: http://dx.doi.org/10.1175/MWR-D-14-00050.1

Publisher: American Meteorological Society

All outputs in CentAUR are protected by Intellectual Property Rights law, including copyright law. Copyright and IPR is retained by the creators or other copyright holders. Terms and conditions for use of this material are defined in the End User Agreement.

www.reading.ac.uk/centaur 
Central Archive at the University of Reading

Reading's research outputs online 


\title{
${ }^{\ominus}$ The Effect of the Equivalent-Weights Particle Filter on Dynamical Balance in a Primitive Equation Model
}

\author{
Melanie Ades And Peter Jan VAn LeEuwen \\ Department of Meteorology, University of Reading, Reading, United Kingdom
}

(Manuscript received 5 February 2014, in final form 4 September 2014)

\begin{abstract}
The disadvantage of the majority of data assimilation schemes is the assumption that the conditional probability density function of the state of the system given the observations [posterior probability density function (PDF)] is distributed either locally or globally as a Gaussian. The advantage, however, is that through various different mechanisms they ensure initial conditions that are predominantly in linear balance and therefore spurious gravity wave generation is suppressed.

The equivalent-weights particle filter is a data assimilation scheme that allows for a representation of a potentially multimodal posterior PDF. It does this via proposal densities that lead to extra terms being added to the model equations and means the advantage of the traditional data assimilation schemes, in generating predominantly balanced initial conditions, is no longer guaranteed.

This paper looks in detail at the impact the equivalent-weights particle filter has on dynamical balance and gravity wave generation in a primitive equation model. The primary conclusions are that (i) provided the model error covariance matrix imposes geostrophic balance, then each additional term required by the equivalent-weights particle filter is also geostrophically balanced; (ii) the relaxation term required to ensure the particles are in the locality of the observations has little effect on gravity waves and actually induces a reduction in gravity wave energy if sufficiently large; and (iii) the equivalent-weights term, which leads to the particles having equivalent significance in the posterior PDF, produces a change in gravity wave energy comparable to the stochastic model error. Thus, the scheme does not produce significant spurious gravity wave energy and so has potential for application in real high-dimensional geophysical applications.
\end{abstract}

\section{Introduction}

Dynamical balance is an inherent part of the majority of geophysical model equations and plays a particularly important role in numerical weather prediction (Gill 1982). A model's dynamical balances are defined physical relationships between different model variables that can cause serious issues with the quality of model predictions if they are not maintained to a certain degree (Daley 1991). In particular, it is important that the initial condition is near balanced in order to avoid the proliferation of gravity waves throughout a model forecast (Lorenc 2003b).

๖ Denotes Open Access content.

Corresponding author address: Melanie Ades, Dept. of Meteorology, University of Reading, Earley Gate, P.O. Box 243, Reading RG6 6BB, United Kingdom.

E-mail: m.ades@reading.ac.uk
In numerical weather prediction the initial conditions for forecasts are generated using data assimilation. Data assimilation is the process of estimating the probability density function (PDF) of the state of a system by combining often noisy, incomplete observations with information from numerical predictions. There are two main classes of data assimilation methods that are currently in use in operational centers: variational methods and ensemble Kalman filters (EnKFs). Variational methods (Lorenc 1986; Le Dimet and Talagrand 1986; Talagrand and Courtier 1987; Courtier and Talagrand 1987) enforce the linear balance relations through a background error covariance matrix, which represents uncertainty in the information from the numerical predictions. The dynamical balance relationships are incorporated in the structure and formulation of the background error covariance matrix and this leads to an initial condition that is predominantly in balance (Lorenc et al. 2000; Lorenc 2003a; Bannister 2008).

The ensemble Kalman filter (Evensen 1994; Burgers et al. 1998) and its variants (Bishop et al. 2001; Anderson 2001; Whitaker and Hamill 2002) use the information 
from an ensemble of model runs to formulate a forecast error covariance matrix. Since the model runs will all produce a nearly balanced state at observation time, this forecast error covariance matrix should also ensure initial conditions that are close to linearly balanced. Unfortunately, spatial localization is necessary in these ensemble methods to avoid spurious long-range correlations and to increase the effective ensemble size. This localization disrupts the enforced relationships between variables (Houtekamer and Mitchell 2005) and means that balanced initialization may be required as an additional step (Buehner et al. 2010).

Although both these classes of data assimilation methods ultimately lead to near-balanced initial conditions, they produce a single analysis model state with an associated analysis error covariance matrix that gives either the local or global uncertainty of the estimate. The underlying assumption is that the conditional PDF for the current state of the system given the observations, known as the posterior PDF, can be represented by a unimodal distribution close to a Gaussian. The presently popular hybrid methods (e.g., Liu et al. 2008) also make this assumption. Historically, this conjecture was justified since the coarse resolution of the models meant the largescale linear flow dominated leading to a Gaussian posterior PDF. As the models have become more nonlinear and grid resolution has increased, however, the appropriateness of this approximation becomes questionable.

Particle filters are a third class of data assimilation methods that do allow for the representation of potentially multimodal posterior PDFs. They portray the information from previous numerical predictions by an ensemble of model runs or particles that are then weighted by their proximity to the observations. The difficulty is that these weights can vary widely, particularly with large numbers of observations, leading to the representation of the posterior PDF by a single and generally uninformative particle (Snyder et al. 2008). This is known as filter degeneracy and considerable research has been undertaken to try and alleviate the issue [see Van Leeuwen (2009) for a review for the geosciences].

The basic formulation of particle filters can be adapted through the use of proposal densities. There is great freedom in how proposal densities can be chosen and many different variants have been explored (Doucet et al. 2001; Morzfeld et al. 2012; Papadakis et al. 2010; Weare 2009). The equivalent-weights particle filter (Van Leeuwen 2010) uses two different proposal densities to ensure samples from the posterior that are in the locality of the observations and for which the majority contribute information on the posterior PDF. The specific proposal densities chosen to ensure these properties result in changes to the model state beyond those already determined by the model equations. The size of these changes can sometimes be larger than the modification to the state induced by just applying the deterministic model equations. Since dynamical balances are an integral part of the deterministic model equations, any change in state because of the deterministic model will be predominantly in balance. The question addressed by this article is what impact the additional terms required by the equivalentweights particle filter will have on a model's dynamical balances and whether it will consequently introduce spurious gravity waves in model forecasts.

The article is structured as follows: Section 2 provides an overview of the basic formulation of particle filters and the manner in which this is adapted by the equivalent-weights particle filter. In particular, it focusses on the displacement in state space required by the choice of proposal density. The impact on dynamical balances is assessed using a primitive equation model described in section 3. In section 4 , the relationship between the equivalent-weights particle filter and dynamical balances is discussed. The effect this has on the representation of the posterior PDF is shown in section 5, and this is followed by a more detailed examination of the effect on gravity waves in section 6. Finally, conclusions are stated in section 7.

\section{Equivalent-weights particle filter}

\section{a. Particle filters}

Particle filters are based on two principles: Bayes's theorem and the Monte Carlo simulation of PDFs. In the Monte Carlo simulation, a PDF is represented by a series of random draws or particles. In this case, it is the information coming from previous numerical predictions, or the prior PDF, which is represented by a set of delta functions centered at individual model states. If the model state of particle $i$ at time $n$ is represented by $\mathbf{x}_{i}^{n}$, then this equates to

$$
p\left(x^{n}\right) \approx \frac{1}{N} \sum_{i=1}^{N} \delta\left(\mathbf{x}_{i}^{n}-\mathbf{x}^{n}\right) .
$$

The observation information is included in this Monte Carlo representation of the model state using Bayes's theorem:

$$
p\left(\mathbf{x}^{n} \mid \mathbf{y}^{n}\right)=\frac{p\left(\mathbf{y}^{n} \mid \mathbf{x}^{n}\right) p\left(\mathbf{x}^{n}\right)}{p\left(\mathbf{y}^{n}\right)}
$$

Together, the two lead to the posterior PDF $\left[p\left(\mathbf{x}^{n} \mid \mathbf{y}^{n}\right)\right]$ being represented by the weighted sum of delta functions:

$$
\begin{gathered}
p\left(\mathbf{x}^{n} \mid \mathbf{y}^{n}\right)= \\
w_{i=1}^{N}=\frac{p\left(\mathbf{y}_{i}^{n} \mid \mathbf{x}_{i}^{n}\right)}{\sum_{k=1}^{N} p\left(\mathbf{x}_{i}^{n}-\mathbf{x}^{n}\right),} .
\end{gathered}
$$


Here, $\mathbf{y}^{n}$ is the observation vector at time $n$, and $p\left(\mathbf{y}^{n} \mid \mathbf{x}_{i}^{n}\right)$ is called the likelihood, since the probability of observing $\mathbf{y}^{n}$ given the model state $\mathbf{x}_{i}^{n}$ is an indication of how likely that observation is given the model state. In effect, the weight of each particle is related to how close the model state of that particle is to the observation vector. Filter degeneracy occurs when insufficient particles are close to the observation at analysis time. This means the normalized likelihood of one particle can dominate the weights and the posterior PDF then effectively becomes represented by this single model state.

The above is a very brief overview of the general formulation of particle filters. A full description is beyond the scope of this article, but for more detailed information see Doucet et al. (2001) or Van Leeuwen (2009).

\section{b. Equivalent-weights particle filter}

The equivalent-weights particle filter is an adaptation of this general formulation using proposal densities. It is designed to avoid filter degeneracy while ensuring that the majority of particles are samples from the high probability region of the posterior PDF. An overview of the full scheme is given in the appendix, but for a theoretical justification of the adaptations, see Ades and Van Leeuwen (2013). Here, the relevant equations with relation to dynamical balances and gravity waves are discussed.

\section{1) Relaxation}

There are two key adaptations made in the equivalentweights particle filter. In the basic particle filter, each particle is moved forward in time to the next observation according to the stochastic model equations:

$$
\mathbf{x}_{i}^{j}=f\left(\mathbf{x}_{i}^{j-1}\right)+d \boldsymbol{\beta}_{i}^{j-1}
$$

where $\mathbf{x}_{i}^{j}$ is the model state of particle $i$ at time $j, f(\cdot)$ are the discretized model equations, and $d \boldsymbol{\beta}_{i}^{j-1}$ is the stochastic error representing unknown terms in the model equations. In this case, the additive stochastic error is drawn from the Gaussian distribution $d \boldsymbol{\beta}_{i}^{j} \sim N(0, \mathbf{Q})$, where $\mathbf{Q}$ is the model error covariance.

The first adaptation made in the equivalent-weights particle filter is to apply an additional term to the model (5). This additional term works to provide a small relaxation toward the future observation at time $n$ :

$$
\mathbf{x}_{i}^{j}=f\left(\mathbf{x}_{i}^{j-1}\right)+\mathbf{B}(\tau)\left[\mathbf{y}^{n}-\mathbf{h}\left(\mathbf{x}_{i}^{j-1}\right)\right]+\widehat{d \boldsymbol{\beta}}_{i}^{j-1} .
$$

The factor $\mathbf{B}(\tau)$ is a matrix specifying the strength of the relaxation dependent on the distance in time $\tau$ to the next observation, and $\mathbf{h}\left(\mathbf{x}_{i}^{j-1}\right)$ is a measurement operator that projects the model state onto the observation space, but at time $j-1$. The term $\widehat{d \boldsymbol{\beta}_{i}^{j-1}}$ still represents unknowns in the model equations but is now drawn from $N(0, \hat{\mathbf{Q}})$. This reflects the fact that the model error may now have also potentially changed because of the change in model equation, with new model error covariance $\hat{\mathbf{Q}}$.

As a consequence of this additional term, the weight of each particle is affected. This can lead to the particles already having significant differences in weight, and hence filter degeneracy, before the likelihood is even taken into account (Ades and Van Leeuwen 2013).

\section{2) EQuiVALENT WEIGHTS}

The second adaptation made to the basic particle filter is therefore to ensure the majority of particles all have equivalent weights when estimating the posterior PDF. The relaxation term is applied to the model equations over all but the last time step before the analysis (4). In the final time step the model state of a chosen percentage of particles is set according to

$$
\mathbf{x}_{i}^{n}=f\left(\mathbf{x}_{i}^{n-1}\right)+\alpha_{i} \mathbf{K}\left[\mathbf{y}^{n}-\mathbf{H} f\left(\mathbf{x}_{i}^{n-1}\right)\right]+\tilde{\mathbf{Q}}^{1 / 2} \boldsymbol{\xi}_{i}^{n},
$$

where $\mathbf{K}=\mathbf{Q} \mathbf{H}^{\mathrm{T}}\left(\mathbf{H} \mathbf{Q} \mathbf{H}^{\mathrm{T}}+\mathbf{R}\right)^{-1}$. The matrix $\mathbf{Q}$ is the original model error covariance, $\mathbf{H}$ is a linearization of the measurement operator $\mathbf{h}(\cdot)$, and $\mathbf{R}$ is the observation error covariance matrix. The term $\tilde{\mathbf{Q}}^{1 / 2} \boldsymbol{\xi}_{i}^{n}$ is the stochastic error term. In this case, $\boldsymbol{\xi}_{i}^{n}$ comes from a mixture density and the use of $\tilde{\mathbf{Q}}^{1 / 2}$ is representative of the fact that the proposal transition density has again changed. The definition of $\alpha_{i}$ is given in the appendix. This choice of model evolution ensures equivalent weights for the specified percentage of particles. The remaining particles will have a smaller weight and so will be abandoned, returning as duplicates of the kept particles when resampling is applied (Kitagawa 1996).

The theoretical justification of why each particle is chosen in this manner is again contained in Ades and Van Leeuwen (2013).

\section{c. Discussion}

It is widely recognized that geophysical models are an approximation to the truth and so stochastic error is necessary to represent unknowns in the model equations. Although the addition of stochastic model error will have an impact on the gravity waves, it is assumed that the stochastic error term is sufficiently small that in general the balances are preserved. The equivalentweights particle filter requires extra terms to be added to the deterministic model equations in addition to the stochastic error term. In previous papers, the size of the additional relaxation term in (6) has been kept smaller than the stochastic error term (Ades and Van Leeuwen 2013, 2014), the implicit assumption being that it would not therefore have an increased impact on gravity waves 
beyond that induced by the model error. The validity of this assumption is now investigated in section $6 \mathrm{c}$.

The additional term required in the equivalentweights step (7) is of more interest. The factor $\alpha_{i}$ in this term dictates the change in model state required by each particle in order to ensure the weights of the majority of particles are equivalent. It was found with both the Lorenz 63 system of equations (Ades and Van Leeuwen 2013) and the barotropic vorticity equation (Ades and Van Leeuwen 2014) that this often led to a change in model state comparable to or larger than that induced by the deterministic model equations. The concern is whether this change causes the introduction of sufficient additional gravity waves to destroy the dominance by the dynamical balances (examined in section $6 \mathrm{~d}$ ). It should be noted that, to a rough approximation, variational and EnKF-based methods would enforce similarsized changes in model state in the primitive equation model, as will be shown later in this article.

\section{Primitive equation model}

\section{a. Single-layer ocean model}

To answer the questions raised on dynamical balances, a single-layer primitive equation model is used. The model is based on horizontal momentum equations and a continuity equation and mimics a wind-driven ocean as a reduced-gravity system:

$$
\begin{aligned}
\frac{\partial u}{\partial t}+u \frac{\partial u}{\partial x}+v \frac{\partial u}{\partial y}-f v+g^{\prime} \frac{\partial h}{\partial x} & =\frac{\tau^{(x)}}{\rho h}-A \Delta u \\
\frac{\partial v}{\partial t}+u \frac{\partial v}{\partial x}+v \frac{\partial v}{\partial y}+f u+g^{\prime} \frac{\partial h}{\partial y} & =-A \Delta v, \\
\frac{\partial h}{\partial t}+\frac{\partial}{\partial x}(h u)+\frac{\partial}{\partial y}(h v) & =0
\end{aligned}
$$

where $\Delta u=\nabla^{2} u$ and similarly for $v$. The numerical model used is a discretization of (8) and calculates perturbations in the depth of the layer $e$ around a fixed depth $H=500 \mathrm{~m}$. The total depth of the layer is given by $h=H+e$, and a velocity field $(u, v)$ is present over the two-dimensional domain. The reduced gravity is represented by $g^{\prime}$ and is chosen equal to $0.005 \times 9.81 \mathrm{~s}^{-2}$. A $\beta$-plane approximation, $f=f_{0}+\beta y$, is used, where $f_{0}=1 \times$ $10^{-4} \mathrm{~s}^{-1}$ is chosen to be at the center of the domain in the meridional direction and $\beta=2 \times 10^{-11}(\mathrm{~ms})^{-1}$. A wind stress $\tau^{(x)} /(\rho h)$ is applied across the domain that induces an eastward flow in the center, where $f=f_{0}$, and a westward flow in the north and south. This, together with the pressure gradient terms $g^{\prime} \partial h / \partial x$ and $g^{\prime} \partial h / \partial y$, drives the flow in the system. The dissipation terms $(-A \Delta u$ and $-A \Delta v)$ are also included, where $A=100 \mathrm{~m}^{2} \mathrm{~s}^{-1}$, and represent momentum sinks to unresolved scales. Stochastic error $d \boldsymbol{\beta}=\left(d \beta_{u}, d \beta_{v}\right.$, $\left.d \beta_{h}\right)^{\mathrm{T}}$ is added to the numerical approximation of the deterministic model (8) at each time step representing unknown and subgrid-scale processes that influence the resolved scales.

The equations are solved for $u, v$, and $e$ over a domain of 100 points in the zonal and 200 points in the meridional, so the system has a dimension of 60000 . The grid spacing is $\Delta x=10 \mathrm{~km}, \Delta y=10 \mathrm{~km}$, and so in physical space the domain is $2000000 \mathrm{~km}^{2}$. The space discretization is on an Arakawa $\mathrm{C}$ grid and a zero flow boundary condition is imposed, with $u=v=0$ and $\partial h / \partial n=0$, with $n$ the outward pointing vector at all the boundaries. The time discretization used in the majority of time steps is a leapfrog scheme with $\Delta t=50 \mathrm{~s}$. A forward Euler scheme is used for the initial time step, the time step immediately following an observation analysis time, and every fiftieth time step. The fiftieth time step Euler scheme is introduced to avoid interference from the computational mode in the leapfrog scheme. The stochastic term was implemented using the Euler-Maruyama scheme in all cases.

This single-layer primitive equation model was used since it is a relatively simple model that incorporates both gravity waves and dynamical balances. The parameters can be chosen such that the model either represents the atmosphere or ocean. Both would be equally appropriate to assess the impact of the equivalentweights particle filter, since the focus of this article is on the dynamics of the gravity waves rather than on the physics of the system. Here, the parameters ensure that the model is representative of an ocean, since this causes a longer dispersion time for any spuriously introduced gravity waves. This leads to greater clarity when attributing any additional gravity wave energy to the constituent parts of the data assimilation process.

\section{b. Balances and gravity waves}

The balance prevalent in the primitive equation model is geostrophic balance:

$$
\begin{aligned}
& f u=-g^{\prime} \frac{\partial h}{\partial y} \\
& f v=g^{\prime} \frac{\partial h}{\partial x} .
\end{aligned}
$$

Figure 1 shows the $u$ field at day 200, the equivalent geostrophic $u$ flow, calculated via

$$
u=-\frac{g^{\prime}}{f} \frac{\partial h}{\partial y},
$$

and the normalized absolute percentage difference between the two fields, which is the unbalanced, ageostrophic 


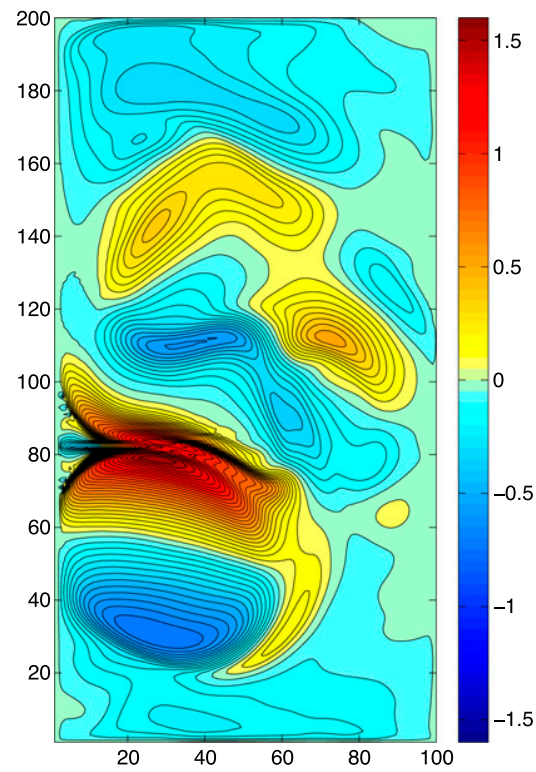

(a) $u$ velocity $\left(m s^{-1}\right)$

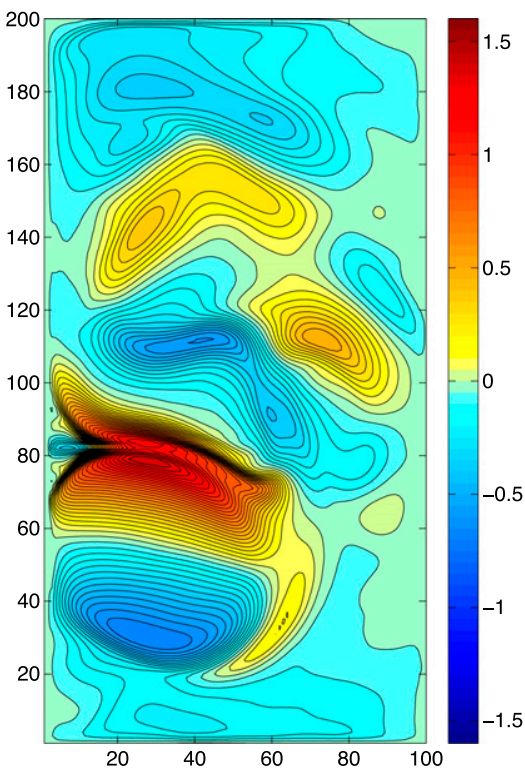

(b) $u$ geostrophic velocity $\left(m s^{-1}\right)$

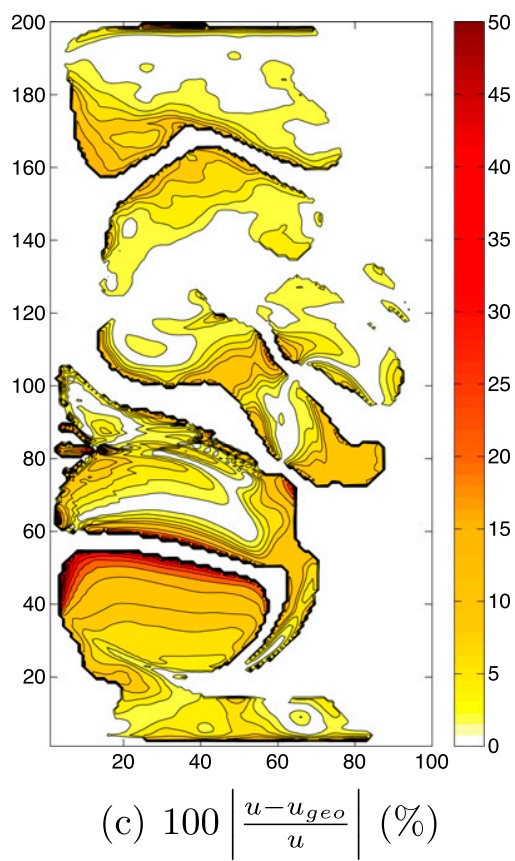

FIG. 1. (a) The actual $u$ field at day 200 of the stochastic primitive equation model compared to (b) the equivalent $u$ field that is in geostrophic balance and (c) the absolute normalized percentage difference between the two. Where values in the actual $u$ field were $<0.1 \mathrm{~m} \mathrm{~s}^{-1}$, the percentage difference was set to zero to avoid erroneous high differences caused by the normalization. It is clear that the ageostrophic flow can be as high as $50 \%$ of the actual flow where there are steep gradients that are not in geostrophic balance.

flow. While the majority of the flow is close to geostrophic, the ageostrophic flow can be as much as $50 \%$ of the actual flow, generally where there are steep gradients in the pressure field.

Gravity waves are also present in the system and these will closely adhere to the gravity wave dispersion relationship

$$
\omega^{2}=f_{0}^{2}+g^{\prime} H\left(k^{2}+l^{2}\right)
$$

where $\omega=2 \pi f$ represents the angular wave frequency and $k=2 \pi \hat{k}$ and $l=2 \pi \hat{l}$ are the angular wavenumbers in the $x$ and $y$ directions, respectively. Here, $f$ is the frequency and $\hat{k}$ and $\hat{l}$ are the wavenumbers per unit distance.

\section{c. Twin experiments}

The twin experiments were performed using a truth run from the stochastic model. The initial condition for the truth run was generated by running the deterministic model forward in time from zero initial conditions until day 200 when chaotic-like behavior was observed. This was then perturbed by random error $d \boldsymbol{\beta}_{\text {initial }} \sim N\left(0, V_{x} \overline{\mathbf{Q}}\right)$ to give the initial conditions for the particles. The term $V_{x}=5000$ was found to give the appropriate perturbations of approximately $10 \%$ of the initial model state, which led to a sufficient spread in the initial condition. The high number for $V_{x}$ is necessary in order to counteract the scaling induced by the formulation of the matrix $\overline{\mathbf{Q}}$ (see below). The random error $d \boldsymbol{\beta} \sim N(0, \mathbf{Q})=N\left(0, V_{\beta} \overline{\mathbf{Q}}\right)$, used for both the truth run and the particles, was generated using a value of $V_{\beta}=$ $0.05^{2} \Delta t$, which led to a $\|d \boldsymbol{\beta}\|$ that was approximately $10 \%$ of $\|f(\mathbf{x})\|$ in the $u$ and $v$ fields and $5 \%$ in the $e$ field. Perturbations of approximately $10 \%$ were used since this is the size of typical error in the atmospheric forcing for ocean models (Brodeau et al. 2010). The same correlation matrix $\overline{\mathbf{Q}}$ was used for both the initial condition perturbations and the model error. The geostrophic balance relationships and boundary conditions between the variables were maintained in the perturbations by using the control variable transforms $\mathbf{U}$ and $\tilde{\mathbf{U}}$ to form the correlation matrix $\overline{\mathbf{Q}}$, so that $\overline{\mathbf{Q}}=\mathbf{U} \tilde{\mathbf{U}} \overline{\mathbf{Q}}_{\tilde{\psi}} \tilde{\mathbf{U}}^{\mathrm{T}} \mathbf{U}^{\mathrm{T}}$. The quantity $\mathbf{U}$ transforms the streamfunction $\psi$ into $u, v$, and $h$ and ensures the correct geostrophic balances (see section 4 for more details). The transform $\tilde{\mathbf{U}}$ takes the streamfunction in the spectral domain to the streamfunction in the physical domain. The sine transform was used since it conserves the no flow boundary conditions. The correlation matrix $\overline{\mathbf{Q}}_{\tilde{\psi}}$ is now specified in Fourier space and is chosen to be a diagonal matrix. A Gaussian variance spectrum was used to specify the correlations with a length scale in wavenumber space of $1 \times 10^{-5} \mathrm{~m}^{-1}$. This is equivalent to waves in physical space with a wavelength of $\lambda=50 \mathrm{~km}$. The Gaussian correlation function 
was chosen since it is simple to compute and the length scale ensures model error perturbations that are larger than the grid scale of $10 \mathrm{~km}$, but smaller than the length scale of the dominant features. This size of model error was specifically chosen since the stochastic error in the equations represents inaccuracies in the deterministic model when compared to the truth arising from subgridscale processes and physics.

Observations were generated as follows. Model states were taken of the perturbation in sea surface height $e$ only, where $h=H+e$, from the truth run every 10 days for the full 120-day model run and every 20 days for the gravity wave experiments. The observations were created by sampling the $e$ field every $30 \mathrm{~km}$ and perturbing the values by observation error taken from $N(0, \mathbf{R})$, where $\mathbf{R}=V_{y} \mathbf{l}$. The term $V_{y}$ is a scalar with values of $(5 \mathrm{~m})^{2}$ corresponding to $h$. Since the model is a reducedgravity model, observation errors in the measurement at the top of the layer of approximately $2.5 \mathrm{~cm}$ correspond to perturbations in the thickness of $h$ of $5 \mathrm{~m}$. The observation errors, pattern, and time spacing for the full model run were chosen to provide a crude approximation to the actual observing patterns of radar altimeter satellites, which observe the height of the sea surface with centimeter accuracy.

The equivalent-weight parameters $\gamma_{U}, \gamma_{N}$, and $\epsilon$ were set equal to $10^{-5}, 10^{-5}$, and $0.001 / N$, respectively (see the appendix). The matrices $\hat{\mathbf{Q}}$ and $\tilde{\mathbf{Q}}$ [see (6) and (7), respectively] were set equal to $\mathbf{Q}$. The factor $\mathbf{B}(\tau)$ in (6) was chosen to be

$$
\mathbf{B}(\tau)=b p(\tau) \mathbf{Q} \mathbf{H}^{\mathrm{T}} \mathbf{R}^{-1},
$$

where $b=50$ and $p(\tau)=\tau$, with $\tau=0$ at the time of the previous observation and increasing linearly to $\tau=1$ at the new observation time. There were 24 particles used for all the experiments, and $100 \%$ of the particles were retained in the equivalent-weights step, since this gives the largest value of the equivalent-weights term $\left\|\alpha_{i} \mathbf{K}\left[\mathbf{y}^{n}-\mathbf{H} f\left(\mathbf{x}_{i}^{n-1}\right)\right]\right\|[$ see (7)].

\section{Balances in the equivalent-weights particle filter}

There are four terms that have the potential to disrupt both the approximate dynamical balances given in (9) and the nonlinear balances in the system: the terms $\mathbf{B}(\tau)\left[\mathbf{y}^{n}-\mathbf{h}\left(\mathbf{x}_{i}^{j-1}\right)\right]$, and $\widehat{d \boldsymbol{\beta}}_{i}^{j-1}$ in (6) and the terms $\alpha_{i} \mathbf{K}\left[\mathbf{y}^{n}-\mathbf{H} f\left(\mathbf{x}_{i}^{n-1}\right)\right]$ and $\tilde{\mathbf{Q}}^{1 / 2} \boldsymbol{\xi}_{i}^{n}$ from (7).

In the truth run of the twin experiment, the truth is run forward in time according to (5). To generate the sample $d \boldsymbol{\beta}_{i}^{j-1} \sim N(0, \mathbf{Q})$, it is standard practice to first generate a sample $\boldsymbol{\xi}_{i}^{j-1} \sim N(0, \mathbf{I})$ and then to multiply this by $\mathbf{Q}^{1 / 2}$, so that $d \boldsymbol{\beta}_{i}^{j-1}=\mathbf{Q}^{1 / 2} \boldsymbol{\xi}_{i}^{j-1}$. Similarly the sample of random error from the relaxation transition density given by (6) is generated using $\widehat{d \boldsymbol{\beta}}_{i}^{j-1}=\hat{\mathbf{Q}}^{1 / 2} \hat{\boldsymbol{\xi}}_{i}^{j-1}$, where $\boldsymbol{\xi}_{i}^{j-1} \sim N(0, \mathbf{I})$. In this article $\hat{\mathbf{Q}}=\mathbf{Q}$, hence the random error used in the true model equations and that used for the relaxation model equations is drawn from the same distribution. This term in the relaxation model equation will therefore produce gravity wave activity in a comparable manner to the stochastic term in the truth and so the gravity wave energy will be similar to that in the truth. The effect of different permutations of this stochastic error on gravity wave energy is studied in detail in section 6a. The random error added under the equivalent-weights step is $\tilde{\mathbf{Q}}^{1 / 2} \boldsymbol{\xi}_{i}^{n}$, where now the random error $\boldsymbol{\xi}_{i}^{n}$ is drawn from a mixture density as detailed in the appendix. The amplitude is significantly lower than that of the model error, so no extra disturbances to the gravity wave energy is expected to arise from this term.

The relaxation matrix $\mathbf{B}(\tau)$ is given in (12), and the equivalent-weights term is expanded to

$$
\alpha_{i} \mathbf{Q H}^{\mathrm{T}}\left(\mathbf{H Q H}^{\mathrm{T}}+\mathbf{R}\right)^{-1}\left[\mathbf{y}^{n}-\mathbf{H} f\left(\mathbf{x}_{i}^{n-1}\right)\right] .
$$

Common to all four of the additional perturbations is the multiplication by $\mathbf{Q}$ or $\mathbf{Q}^{1 / 2}$. The matrix

$$
\mathbf{Q}=V_{\beta} \overline{\mathbf{Q}}=V_{\beta} \mathbf{U} \tilde{\mathbf{U}} \overline{\mathbf{Q}}_{\tilde{\psi}} \tilde{\mathbf{U}}^{\mathrm{T}} \mathbf{U}^{\mathrm{T}}
$$

is defined for the primitive equation model via the control variable transforms $\mathbf{U}$ and $\tilde{\mathbf{U}}$. The term $\mathbf{U}$ takes the streamfunction $\psi$ and transforms it into $u, v$, and $h$ via

$$
u=-\frac{\partial \psi}{\partial y}, \quad v=\frac{\partial \psi}{\partial x}, \quad h=\frac{f_{0}}{g^{\prime}} \psi .
$$

Given that

$$
h=\frac{f_{0}}{g^{\prime}} \psi \Rightarrow \psi=\frac{g^{\prime}}{f_{0}} h,
$$

the geostrophic balance relationships

$$
\begin{gathered}
u=-\frac{g^{\prime}}{f_{0}} \frac{\partial h}{\partial y} \\
v=\frac{g^{\prime}}{f_{0}} \frac{\partial h}{\partial x}
\end{gathered}
$$

are enforced by this transformation. For example, given a vector $\left(u_{b} v_{b} h_{b}\right)^{\mathrm{T}}=\mathbf{Q}(u v h)^{\mathrm{T}}=V_{\beta} \mathbf{U} \tilde{\mathbf{U}} \overline{\mathbf{Q}}_{\tilde{\psi}} \tilde{\mathbf{U}}^{\mathrm{T}} \mathbf{U}^{\mathrm{T}}(u v h)^{\mathrm{T}}$, then $\mathbf{U}^{\mathrm{T}}$ first takes the vector in physical space and transforms it into the streamfunction in physical space, $\tilde{\mathbf{U}}^{\mathrm{T}}$ then transforms the resultant vector into the equivalent streamfunction in spectral space, $\overline{\mathbf{Q}}_{\tilde{\psi}}$ introduces the appropriate correlations to the spectral streamfunction vector, and $\tilde{\mathbf{U}}$ transforms the vector back into the 


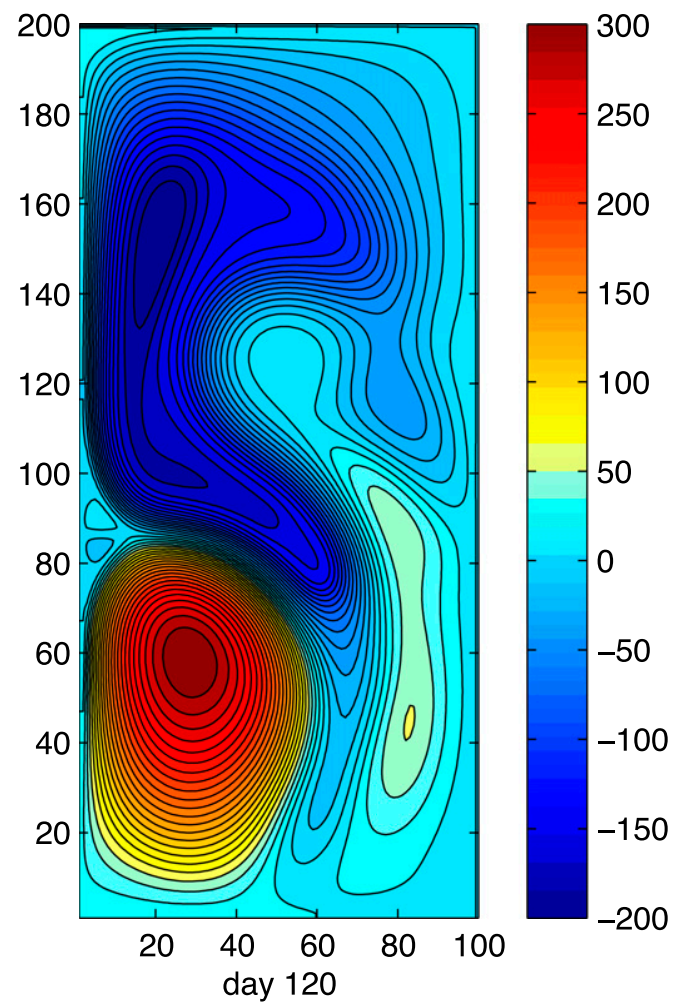

(a) True model state

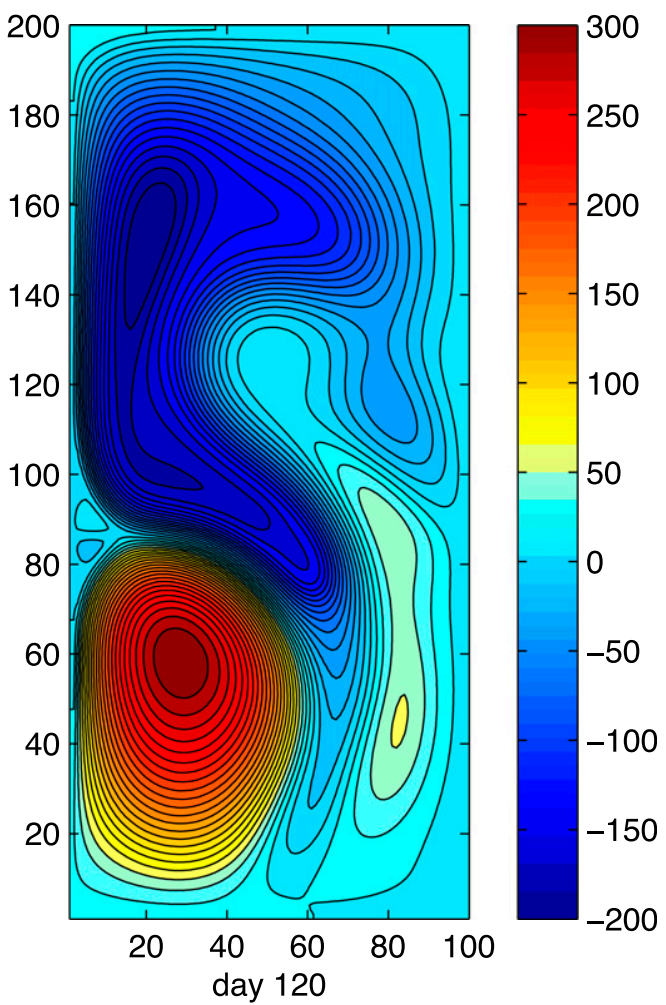

(b) Mean of 24 particles

FIG. 2. (a) The true model state for $e$ in the primitive equation after 120 days compared to (b) the mean of 24 particles under the conditions given in section $3 \mathrm{c}$, with a contour interval of $17.5 \mathrm{~m}$. It is clear that the equivalentweights particle filter provides a close match to the mean, although small differences are evident.

streamfunction in physical space before finally $\mathbf{U}$ takes the streamfunction vector in physical space and transforms it into the appropriate values of $u_{b}, v_{b}$, and $h_{b}$ via (15). It is this final transformation that ensures the vector $\left(u_{b} v_{b} h_{b}\right)^{\mathrm{T}}$ is in geostrophic balance as given by (17).

Since the multiplication by the matrix $\mathbf{Q}$ or $\mathbf{Q}^{1 / 2}$ ensures geostrophic balance through the use of the $\mathbf{U}$ transform, each of the additional perturbations (as well as the addition of random error) should in itself be a geostrophically balanced model state. However, there is no guarantee that these separately geostrophically balanced model states added to $f\left(\mathbf{x}_{i}^{j-1}\right)$ or $f\left(\mathbf{x}_{i}^{n-1}\right)$ will produce a model state for $\mathbf{x}_{i}^{j}$ or $\mathbf{x}_{i}^{n}$ that is without additional unbalanced perturbations leading to the introduction of spurious gravity waves.

\section{Representation of the posterior PDF}

The first test to judge how well the equivalent-weights particle filter is able to perform in a model that includes balances is to compare the mean of the particles to the true model state used in the twin experiments. After 120 days (12 observation analysis times), it was found that the mean of the equivalent-weights particle filter provided a good match to the truth with the equivalentweight parameter values used (Fig. 2 shows this for $e$, but it was also found for the $u$ and $v$ variables). This indicates that the equivalent-weights particle filter is able to produce representative model states in a highdimensional model with multiple variables with complex relationships. Since the focus of this article is on the effect of the equivalent-weights particle filter on dynamical balance, a full consideration of the performance of the scheme is not given here. For an in-depth discussion on the behavior of the scheme in a highdimensional model, see Ades and Van Leeuwen (2014).

These results could indicate that the scheme is not causing an excessive number of spurious gravity waves, since this would lead to unbalanced model states being generated by individual particles and a mean unrepresentative of the truth. It is also possible, however, that there is simply not the same displacement in state space under the equivalent-weights step as was generated by the Lorenz 63 (Ades and Van Leeuwen 2013) or 
TABLE 1. The $l^{2}$ norms $\left(\|\cdot\|_{i, n}=\mathbb{E}_{i, n}\left[\sqrt{\sum_{k=1}^{K}(\cdot)_{k}^{2}}\right]\right.$, where $K$ is the dimension of $e$ ) of the additional terms required by the equivalent-weights particle filter compared to the change in model state due to the deterministic model equations. The deterministic change in state space $[f(\mathbf{x})]$, stochastic error $(d \boldsymbol{\beta})$, and relaxation term $\left\{\mathbf{r}=\mathbf{B}(\tau)\left[\mathbf{y}^{n}-\mathbf{h}\left(\mathbf{x}_{i}^{n-1}\right)\right]\right\}$ have been taken in the second to last time step before an observation. The equivalent-weights term $\left\{\right.$ ew $\left.=\alpha_{i} \mathbf{K}\left[\mathbf{y}^{n}-\mathbf{H} f\left(\mathbf{x}_{i}^{n-1}\right)\right]+\tilde{\mathbf{Q}}^{1 / 2} \boldsymbol{\xi}_{i}^{n}\right\}$ has been taken for each analysis time. Apart from the initial condition perturbation (ic), all norms have been averaged across all analysis times $(n)$ and all particles $(i)$.

\begin{tabular}{lllc}
\hline \hline & \multicolumn{1}{c}{$u$} & \multicolumn{1}{c}{$v$} & $e$ \\
\hline$\|f(\mathbf{x})\|_{i, n}$ & $8.6 \times 10^{-4}$ & $8.6 \times 10^{-4}$ & 0.09 \\
$\|d \boldsymbol{\beta}\|_{i, n}$ & $8.3 \times 10^{-5}$ & $8.5 \times 10^{-5}$ & 0.005 \\
$\|\mathbf{r}\|_{i, n}$ & $2.3 \times 10^{-7}$ & $2.5 \times 10^{-7}$ & $2.7 \times 10^{-5}$ \\
$\|$ ew $\|_{i, n}$ & 0.1 & 0.1 & 13.6 \\
$\|$ ic $\|_{i}$ & 2.6 & 2.6 & 162.4 \\
\hline
\end{tabular}

barotropic vorticity (Ades and Van Leeuwen 2014) models. Table 1 shows the $l^{2}$ norms of the different constituent terms that are implemented as part of the equivalent-weights particle filter. The factor $b=50.0$ in the relaxation matrix $\mathbf{B}(\tau)$ (12) was specifically chosen to ensure that the relaxation term was smaller than the stochastic error, and this is demonstrated in Table 1 . What is also immediately apparent is that the size of the equivalentweights term is more than 100 times the size of the displacement in state space induced by the deterministic model equations when $100 \%$ of particles are retained. However, this is an average over each analysis time, and each particle and is being skewed by a few particles with a large equivalent-weights term. If each individual analysis time is considered independently for a single representative particle, then this number is generally $O(10)$ times larger than the $l^{2}$ norm of the change in model state caused by the deterministic model equations (Table 2). Regardless of which table is considered, using the equivalent-weights particle filter in conjunction with this primitive equation model is causing a substantial change in model state through the application of the equivalent-weights step.

The conclusion can be drawn that the equivalent-weights particle filter is not creating sufficient additional gravity waves in this primitive equation model to create an ensemble mean unrepresentative of the truth, despite the size of the equivalent-weights term. Nonetheless, other models may be more sensitive to changes in model state and the equivalent-weights term is large enough that further examination of the effect on gravity waves is necessary.

\section{Gravity waves in the equivalent-weights particle filter}

The effect of the additional terms on gravity waves was quantified using their known dispersion relationship
TABLE 2. A comparison of the $l^{2}$ norms $\left[\|\cdot\|=\sqrt{\sum_{k=1}^{K}(\cdot)_{k}^{2}}\right.$, where $K$ is the dimension of $e]$ for the relaxation term $\{\mathbf{r}=\mathbf{B}(\tau)$ $\left.\left[\mathbf{y}^{n}-\mathbf{h}\left(\mathbf{x}_{i}^{n-1}\right)\right]\right\}$ and the equivalent-weights term $\left\{\right.$ ew $=\alpha_{i} \mathbf{K}\left[\mathbf{y}^{n}-\right.$ $\left.\left.\mathbf{H} f\left(\mathbf{x}_{i}^{n-1}\right)\right]+\tilde{\mathbf{Q}}^{1 / 2} \boldsymbol{\xi}_{i}^{n}\right\}$ for a specific particle at each analysis time. The $l^{2}$ norms of the deterministic model term $[f(\mathbf{x})]$ and the stochastic error $(d \boldsymbol{\beta})$ have also been included for comparison.

\begin{tabular}{ccccc}
\hline \hline Obs time & $\|f(\mathbf{x})\|$ & $\|d \boldsymbol{\beta}\|$ & $\|\mathbf{r}\|$ & $\|$ ew $\|$ \\
\hline 10 & 0.23 & $5.00 \times 10^{-3}$ & $2.31 \times 10^{-5}$ & 0.74 \\
20 & 0.14 & $5.62 \times 10^{-3}$ & $2.27 \times 10^{-5}$ & 7.89 \\
30 & 0.11 & $5.22 \times 10^{-3}$ & $2.47 \times 10^{-5}$ & 1.33 \\
40 & 0.08 & $5.46 \times 10^{-3}$ & $2.42 \times 10^{-5}$ & 0.85 \\
50 & 0.07 & $5.14 \times 10^{-3}$ & $2.78 \times 10^{-5}$ & 1.27 \\
60 & 0.06 & $4.91 \times 10^{-3}$ & $2.48 \times 10^{-5}$ & 1.27 \\
70 & 0.06 & $5.40 \times 10^{-3}$ & $2.96 \times 10^{-5}$ & 1.79 \\
80 & 0.06 & $5.10 \times 10^{-3}$ & $2.96 \times 10^{-5}$ & 1.78 \\
90 & 0.06 & $5.19 \times 10^{-3}$ & $3.11 \times 10^{-5}$ & 0.95 \\
100 & 0.06 & $5.25 \times 10^{-3}$ & $2.66 \times 10^{-5}$ & 1.67 \\
110 & 0.07 & $5.39 \times 10^{-3}$ & $2.48 \times 10^{-5}$ & 1.90 \\
120 & 0.07 & $5.22 \times 10^{-3}$ & $2.55 \times 10^{-5}$ & 1.38 \\
\hline
\end{tabular}

(11). To calculate the $\omega(\kappa)$ relation from the model simulations, where $\kappa=\sqrt{k^{2}+l^{2}}$, samples were taken of the full model state of $e$ minus 10 grid points around the boundary in the $x$ direction and 20 grid points in the $y$ direction, every hour for 20 days. These samples were then put through a three-dimensional discrete fast Fourier transform (FFT) to calculate the spectrum related to the constituent frequencies of the waves:

$$
\mathbf{X}_{\tilde{\omega}, \tilde{k}, \tilde{l}}=\sum_{t=1}^{T} \sum_{i=1}^{N} \sum_{j=1}^{M} \mathbf{X}_{t, i, j} \varepsilon_{T}^{(t-1)(\tilde{\omega}-1)} \varepsilon_{N}^{(i-1)(\tilde{k}-1)} \varepsilon_{M}^{(j-1)(\tilde{l}-1)},
$$

where

$$
\varepsilon_{N}=\exp \left(\frac{-2 \pi i}{N}\right)
$$

(definition taken from the MATLAB fftn function used to calculate the FFT). The values of $\tilde{\omega}, \tilde{k}$, and $\tilde{l}$ represent the number of waves per 20 days $(\tilde{\omega})$, per $800 \mathrm{~km}(\tilde{k})$, and per $1600 \mathrm{~km}(\tilde{l})$.

The gravity wave dispersion relationship used for this analysis was

$$
\omega^{2}=f_{0}^{2}+g^{\prime} H \kappa^{2},
$$

where $\tilde{\kappa}$ was set equal to $\tilde{\kappa}=(2 \tilde{k})^{2}+\tilde{l}^{2}$, ensuring that it represents wavenumbers over a common distance of $1600 \mathrm{~km}$. However, this meant some of the values of $\tilde{\kappa}$ were duplicates. When this occurred, the corresponding values of the FFT were summed over all possible combinations of $\tilde{k}$ and $\tilde{l}$, leading to that value of $\tilde{\kappa}$.

Figure 3 shows the power spectrum of $\hat{\kappa}=\tilde{\kappa} / 16$ against $\hat{\omega}=\tilde{\omega} / 20$, where $\hat{\kappa}$ is the number of waves per $100 \mathrm{~km}$ 


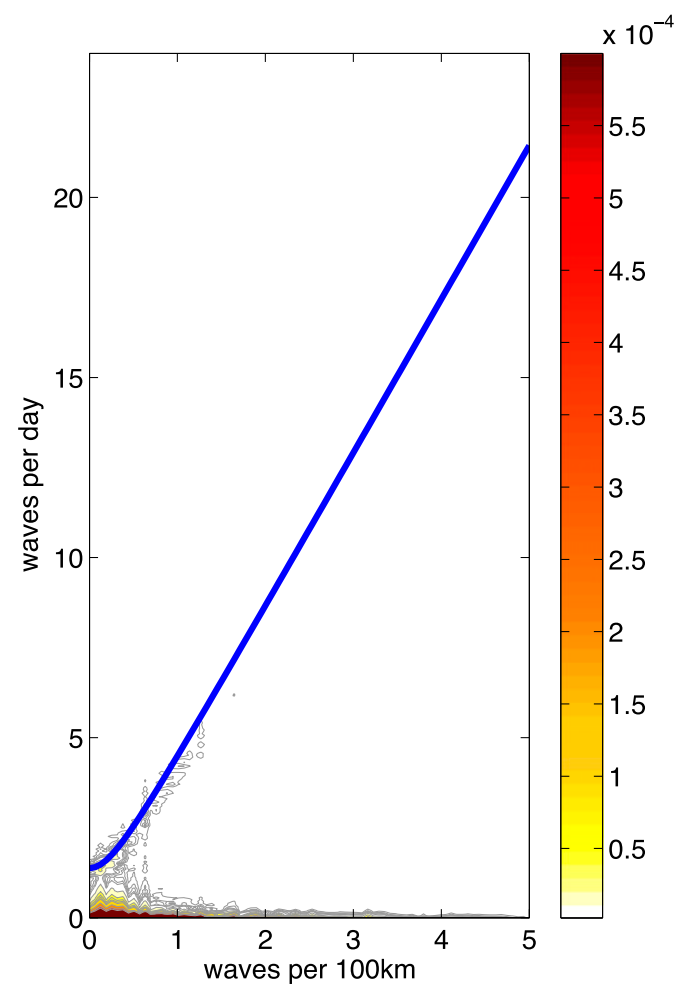

(a) Truth

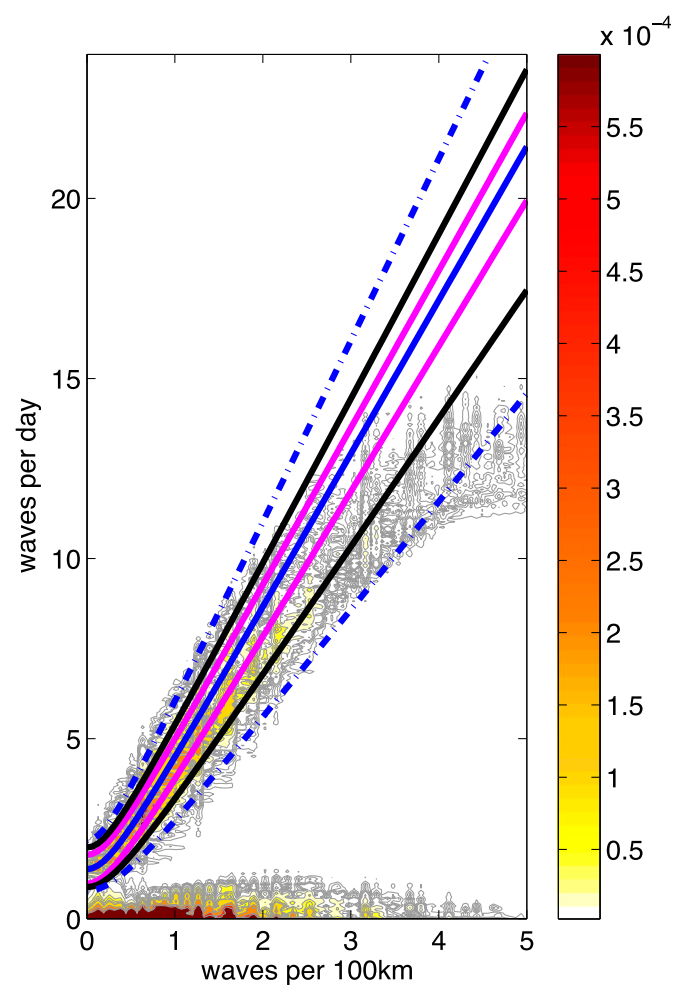

(b) Perturbed initial condition

FIG. 3. (a) The absolute spectrum of the truth run of the model from day 0 to day 20 compared to (b) a particle model run with a perturbed initial condition. To generate the plots, samples were taken of the model state excluding grid points that are $<100 \mathrm{~km}$ from the east-west boundaries and $<200 \mathrm{~km}$ from the north-south boundaries every $30 \mathrm{~min}$. The solid blue line represents the gravity waves dispersion relation, the magenta lines represent band $\mathrm{A}$, the black lines represent band B, and the dashed blue lines represent band C (see text). The contour lines are drawn in gray and so areas that appear predominantly gray are areas with lower energy. The sum of the power spectrum below each of the band lines was taken as the low-frequency motions and above as high-frequency motions. The increased gravity wave energy due to the initial condition perturbation is clear.

and $\hat{\omega}$ is the frequency of waves per day. However, the gravity waves' dispersion relationship relates to the angular frequencies $\kappa=2 \pi \hat{\kappa}$ and $\omega=2 \pi \hat{\omega}$. The values of $f_{0}$, $g^{\prime}$, and $H$ are also in $\mathrm{s}^{-1}, \mathrm{~m} \mathrm{~s}^{-2}$, and $\mathrm{m}$, respectively. To transform them into the correct values per day and per $100 \mathrm{~km}$ requires transforming $f_{0}$ into $\hat{f}_{0}=(24 \times 60 \times 60) f_{0}$ and $g^{\prime} H$ into $\hat{g}^{\prime} \hat{H}=\left[(24 \times 60 \times 60)^{2} / 100000^{2}\right] g^{\prime} H$. This leads to the gravity wave dispersion relationship

$$
\hat{\omega}=\frac{1}{2 \pi} \sqrt{\hat{f}_{0}^{2}+\hat{g}^{\prime} \hat{H}(2 \pi \hat{\kappa})^{2}}
$$

shown as the blue line in Fig. 3.

To quantify the energy associated with gravity waves, bands were chosen with different widths to surround the gravity wave dispersion relation (Fig. 3b). The narrowest band A was chosen to have a width that incorporated the areas of greatest increase, but not the total spread of the energy that could potentially be associated with gravity waves. The band $\mathrm{C}$ was chosen wide enough to ensure that the vast majority of gravity wave energy would be captured, and B formed an intermediate measure. It was assumed that all energy below either band $\mathrm{A}, \mathrm{B}$, or $\mathrm{C}$ could be approximately associated with low-frequency motions such as Rossby wavelike features and nonlinear oscillations of the recirculation cells. Similarly, energy lying above a particular band relates to high-frequency motions such as the stochastic error. The power spectrum was summed over all the points lying within these categories, and the percentage change between different model runs calculated. In general, very little difference was seen between the results from the different bands. The conclusions drawn below refer only to band $\mathrm{B}$, but similar deductions can be made for bands $\mathrm{A}$ and $\mathrm{C}$.

\section{a. Stochastic error}

If the primitive equation model is run deterministically from day $0-20$, without the addition of the stochastic 
error, then there is slight evidence of the gravity wave relationship appearing at longer wavelengths and lowerfrequency waves. However, the majority of the energy can be attributed to slow, large-scale, and balanced motions. Very little difference is seen with the addition of stochastic error (Fig. 3a). The sum of the power spectrum values associated with gravity waves for band $\mathrm{B}$ for the deterministic model run is $4.96 \times 10^{-8}$. In comparison, the stochastic model run has a value of $5.28 \times 10^{-8}$, an increase of $6.5 \%$. As would be expected, the greatest increase is seen in the sum of the power spectrum over all values above the band $\mathrm{B}$ line, which can be attributed to high-frequency motions. This is $5.62 \times 10^{-11}$ for the deterministic model run and $1.74 \times$ $10^{-10}$ for the stochastic model run, an increase of $210 \%$, although still with very low energy values. No real difference is seen in the energy of the low-frequency motions. It is this stochastic model run that is taken as the truth and from which the observations are generated in the twin experiments. It therefore forms the base line to which the model runs for each particle are compared to in the following experiment.

The first experiment to be run was to quantify the effect of using different samples of stochastic error for each particle. This was done so that the impact of the relaxation and equivalent-weights term could be considered in comparison to the difference in gravity wave energy caused by changing the stochastic error. To isolate the effect of the stochastic error, the initial condition was kept the same as the truth for each particle and no relaxation term was applied. Table 3 shows the maximum, mean, and minimum percentage change compared to the truth across the particles for band $\mathrm{B}$ (with associated low- and high-frequency motions). Although on average a $2 \%$ decrease in gravity wave energy occurred, the particles spanned the range between a $4.6 \%$ decrease and a $0.2 \%$ increase. It is to be expected that different permutations of stochastic model error would lead to variations in gravity wave energy over the 20 days, and this is verified by the presence of both positive and negative changes.

\section{b. Initial conditions}

The next experiment was designed to test the impact of using different initial conditions for each of the particles on the gravity wave energy. Similar to the stochastic error experiment, this was undertaken to provide comparative percentage changes for the relaxation and equivalent-weights steps. In the equivalent-weights particle filter, the initial condition for each particle is generated by perturbing the "true" initial condition with random error drawn from $N\left(0, V_{x} \overline{\mathbf{Q}}\right)$ (see section $3 \mathrm{c}$ ). The matrix $\overline{\mathbf{Q}}$ ensures the perturbation is in geostrophic
TABLE 3. The percentage changes in the sum of the power spectrum due to the listed perturbations for band $\mathrm{B}$. The equivalentweights particle filter was run with observations every 20 days and $100 \%$ of the particles were retained to ensure the greatest change in model state at the equivalent-weights step. The random error $d \boldsymbol{\beta}$ for the truth run and each particle of the equivalent-weights particle filter is sampled from $d \boldsymbol{\beta} \sim N\left(0, V_{\beta} \overline{\mathbf{Q}}\right)$, where $V_{\beta}=$ $0.05^{2} \Delta t$, and the initial condition perturbation $d \boldsymbol{\beta}_{\text {initial }} \sim N(0$, $V_{x} \overline{\mathbf{Q}}$ ), where $V_{x} 5000$ (see section 3 c for full details of the parameters). The output of all 24 particles was considered, and the table shows the maximum, mean, and minimum percentage changes observed over the particle values.

\begin{tabular}{llccc}
\hline \hline & & $\begin{array}{c}\text { Low- } \\
\text { frequency } \\
\text { motions }\end{array}$ & $\begin{array}{c}\text { Gravity } \\
\text { waves }\end{array}$ & $\begin{array}{c}\text { High- } \\
\text { frequency } \\
\text { motions }\end{array}$ \\
\hline Stochastic & Max & 0.01 & 0.20 & 2.67 \\
error & Mean & 0.01 & -2.25 & -1.27 \\
Initial condition & Min & 0.0 & -4.58 & -6.05 \\
& Max & 0.76 & 63174 & 152515 \\
Relaxation & Min & 0.07 & 48270 & 118574 \\
& Max & 0.0 & 0.0 & 0.02893 \\
Equivalent & Mean & 0.0 & 0.0 & 0.0 \\
weights & Max & 0.0 & 0.0 & 0.0 \\
& Mean & 0.02 & 1.45 & 1.61 \\
& Min & -0.00 & 0.21 & 0.28 \\
& & & -0.79 & -2.01 \\
\hline
\end{tabular}

balance, and the value of $V_{x}$ is chosen such that the perturbation size is approximately $10 \%$ of the overall field for $u$ and $v$. To judge the impact of this large but geostrophically balanced perturbation on the gravity waves, the power spectrum of each particle without relaxation (Fig. 3b) was compared to the true model run from day 0 to 20 (Fig. 3a). The stochastic error for each particle was fixed to be the same as the true model run so that any changes are specifically related to this initial condition perturbation.

It was found that the initial condition perturbation had an extremely large impact on both the gravity wave energy and the high-frequency motions. Figure $3 b$ shows the spectral energy of the model run when initial condition perturbations are included. A much higher gravity wave energy is visible along the theoretical gravity wave dispersion relation. The bending away from the theoretical dispersion relation at higher wavenumbers is simply related to numerical dispersion of the gravity waves in the model using the leapfrog scheme on a $\mathrm{C}$ grid (see, e.g., Adcroft et al. 1999, his Fig. 5). The average gravity wave energy change across all the particles was a $4.8 \times 10^{4} \%$ increase and an even larger average percentage increase of $11.8 \times 10^{4} \%$ was observed for the high-frequency motions. Despite the fact that the initial perturbation is in geostrophic balance, it leads to a large increase in the amount of gravity wave energy. It should 
be noted at this point that the initial condition perturbation is not unique to the equivalent-weights particle filter and a similar perturbation would be applied to generate the ensemble members in an ensemble Kalman filter (see, e.g., Evensen 2003).

The dissipation rate of the gravity wave energy associated with this initial condition can be observed by comparing the gravity wave energy every 5 days for the true model run in contrast to a model run starting from a perturbed initial condition (Fig. 4). The slow dissipation rate associated with using this single-layer ocean model is evident, and it is not until day 100 that the gravity wave energy approaches the values associated with the true model run. Although the model has a relatively slow dissipation rate, there is still clearly an exponential decrease in gravity wave energy over the first 20 days. The percentage increases because of the perturbed initial condition given above are for a 20-day run of the model. Similar analyses were also carried out for a 5- and 10-day model run to assess whether the higher amount of gravity wave energy over these periods would affect the results. As would be expected, the exact percentage values change; however, the conclusion that the initial condition perturbation leads to a significant increase in gravity wave energy and high-frequency motions remains the same regardless of the number of days chosen.

\section{c. Relaxation proposal density}

To test the effect of applying the relaxation term in the equivalent-weights particle filter, a model run from day 0 to 20 was first generated for each particle with perturbed initial conditions and different stochastic error samples but without the relaxation term. The particles were then run from the same initial conditions with identical samples of model error but now the relaxation term was added. Therefore, any changes in gravity wave energy between the two versions for each particle can be solely attributed to the relaxation toward the future observation.

It was found that the addition of the relaxation term actually had no effect on any part of the spectrum (Table 3 ). This can be attributed to the fact that the size of the relaxation term is considerably smaller than the stochastic error term (Table 2). The same analysis was carried out using days $0-5$ and days $0-10$ and again, no difference was found. As noted in the previous subsection, the initial condition perturbation influence does not dissipate until around day 100 . Hence, the analysis was also carried out comparing the model run with and without relaxation from day 100 to 120 to avoid any potential influence on the results from the initial condition. It was also found that the relaxation term had no effect in this case.

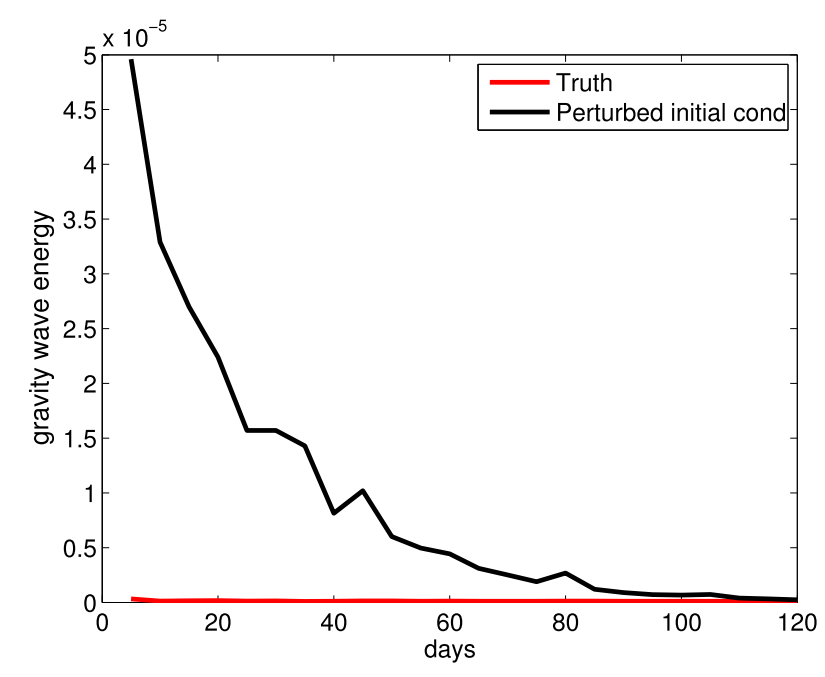

FIG. 4. The gravity wave energy associated with the true model run (red line visible just above the $x$ axis) compared to the gravity wave energy when the initial condition is perturbed (black line). In both runs, the gravity wave energy in band B (see Fig. 3) was calculated for each 5-day period. The exponential decay of the additional gravity wave energy introduced by perturbing the initial condition is evident.

In Ades and Van Leeuwen (2013), it was shown with the barotropic vorticity model that increasing the size of the stochastic error leads to an increase in the size of the relaxation term. A similar approach can be used here to increase the size of the relaxation term. Increasing the stochastic term by a factor of $10^{5}$ leads to a $10^{7}$ increase in the relaxation term. Again, the particles with this increased stochastic error but no relaxation can be compared to the same particles when the now greatly increased relaxation is added. In this case, the results show that the addition of the relaxation term actually led to a decrease in the gravity wave energy in band B for all particles $(-2 \%$ to $-11 \%)$. One hypothesis for why this is occurring is that the relaxation term is actually applying a damping to the propagation of the gravity waves caused by this inflated model error. The slight relaxation toward the fixed observations at every time step reduces the ability of the gravity waves to freely evolve and results in an overall reduction in the gravity wave energy for each particle.

\section{d. Equivalent-weights proposal density}

The size of the equivalent-weights term varies depending on the analysis time chosen. To address the impact of the displacement in state space caused by this term, the day 100 analysis time was used. The day 100 analysis time has a representative $l^{2}$ norm for the equivalent-weights displacement (Table 2), but is sufficiently far from the start of the model run that the 
majority of gravity wave energy caused by the initial condition perturbation should have dispersed.

To assess the impact on gravity waves of the equivalent-weights term, the model state of each particle in the full model run was outputted both before and after the equivalent-weights step at day 100 . These model states were then taken as the initial conditions in a 20-day model run, for which the stochastic error was kept fixed. No relaxation term was applied to ensure that any differences in gravity wave energy are caused only by the change in model state induced by ensuring equivalent weights at day 100 .

On average, a very small increase in gravity wave energy in band $\mathrm{B}$ was observed across all the particles $(0.2 \%$; see Table 3$)$. The changes ranged from a $0.8 \%$ decrease to a $1.5 \%$ increase. Similar results were found when the same assessment was carried out for a 10-day model run $(-1.5 \%$ to $1.7 \%)$ and for a 5 -day model run $(-0.7 \%$ to $1.2 \%)$. Comparing this to the percentage change in gravity waves associated with the different permutations of stochastic error $(-4.6 \%$ to $0.2 \%)$ indicates that this change is of the same order.

To put the size of the equivalent-weights term in context, the typical change in model state of four-dimensional variational data assimilation (4D-Var) or the ensemble members in the EnKF can be approximated by

$$
\mathbf{P}^{f} \mathbf{H}^{\mathrm{T}}\left(\mathbf{H} \mathbf{P}^{f} \mathbf{H}^{\mathrm{T}}+\mathbf{R}\right)^{-1}\left[\mathbf{y}-\mathbf{H}\left(\mathbf{x}_{i}\right)\right] .
$$

To gain a very rough estimate of the size of this term, the assumption is made that $\mathbf{P}^{f}$ is diagonal with entries $\sigma_{x}^{2}$, representing the average variance in each $e$ variable across the ensemble. The observation error variance for $e$ is taken as $V_{y}=\sigma_{y}^{2}=25$ for the diagonal $\mathbf{R}$ matrix, and for this order of magnitude estimate, it is assumed that $e$ is observed at each grid point. It is also necessary to understand the change in model state coming from the factor $\left[\mathbf{y}-\mathbf{H}\left(\mathbf{x}_{i}\right)\right]$. The value of interest is

$$
\begin{aligned}
\left\|\mathbf{y}-\mathbf{H}\left(\mathbf{x}_{i}\right)\right\| & =\sqrt{\operatorname{Tr}\left\{\left[\mathbf{y}-\mathbf{H}\left(\mathbf{x}_{i}\right)\right]\left[\mathbf{y}-\mathbf{H}\left(\mathbf{x}_{i}\right)\right]^{\mathrm{T}}\right\}} \\
& \approx \sqrt{\operatorname{Tr}\left(\mathbf{R}+\mathbf{H} \mathbf{P}^{f} \mathbf{H}^{\mathrm{T}}\right)} .
\end{aligned}
$$

Taking all these approximations together leads to

$$
\begin{gathered}
\left\|\mathbf{P}^{f} \mathbf{H}^{\mathrm{T}}\left(\mathbf{H} \mathbf{P}^{f} \mathbf{H}^{\mathrm{T}}+\mathbf{R}\right)^{-1}\left[\mathbf{y}-\mathbf{H}\left(\mathbf{x}_{i}\right)\right]\right\| \\
\approx \frac{\sigma_{x}^{2}\left(\sigma_{x}^{2}+\sigma_{y}^{2}\right)^{1 / 2}}{\left(\sigma_{x}^{2}+\sigma_{y}^{2}\right)}\left\|\mathbf{I}_{K}\right\|,
\end{gathered}
$$

where $\mathbf{K}$ is the dimension of the $e$ variable, which in this case is 20000 . To evaluate this expression it is additionally necessary to estimate $\sigma_{x}^{2}$. Taking the initial ensemble spread of $\sim O(200)$, and using similar arguments to those given above, this value can be approximated as $\sigma_{x}^{2}=1.4$. This leads to changes in model state due to EnKF or 4D-Var of $O(40)$. Although these are very crude approximations, they can be used to set the change in model state caused by the equivalent-weights term in context. The average change in model state due to the equivalent-weights term is 13.6 (Table 1). Hence, the change in model state of the equivalent-weights particle filter and what could be expected for the EnKF or 4D-Var are roughly comparable for this experiment.

\section{e. Discussion}

The results presented above, both in conjunction with the relaxation and the equivalent-weights proposal densities, would suggest that the additional terms of the equivalent-weights particle filter are not likely to cause significant problems. The question remains as to what extent these results then depend on the specific conditions chosen for the model runs and on the model itself.

It has been noted in previous papers that the equivalent-weights particle filter can be tuned depending on the performance measure of interest (Ades and Van Leeuwen 2013) using the factor $b$ in the relaxation term (12) and the percentage of particles retained under the equivalent-weights step (Ades and Van Leeuwen 2014). The parameters used to test the effect of the equivalent-weights term in this article were artificially chosen to ensure a worst-case scenario. Hence, a high value of $b$ is used and $100 \%$ of particles were retained under equivalent weights, which leads to greater displacement in state space than retaining a smaller percentage of particles. Using these parameter settings, the $l^{2}$ norm of the equivalent-weights term was of the same order of magnitude for the primitive equation as that observed for the barotropic vorticity equation (Ades and Van Leeuwen 2014). However, it is possible that larger changes in model state would be observed if the model was run for longer or if a different model was used.

To judge whether an increase in the size of the equivalent-weights term directly results in an increase in gravity wave energy, the $l^{2}$ norm of the equivalentweights term for each particle was plotted against the corresponding percentage change in gravity wave energy for band B (Fig. 5). No conclusive relationship between the two was observed, which provisionally leads to the conclusion that a larger equivalent-weights term does not necessarily imply a greater increase in gravity wave energy.

This conclusion is drawn by considering particles that have relatively similar-sized equivalent-weights $l^{2}$ norms (0-2.1). The effect of a much greater change to the 


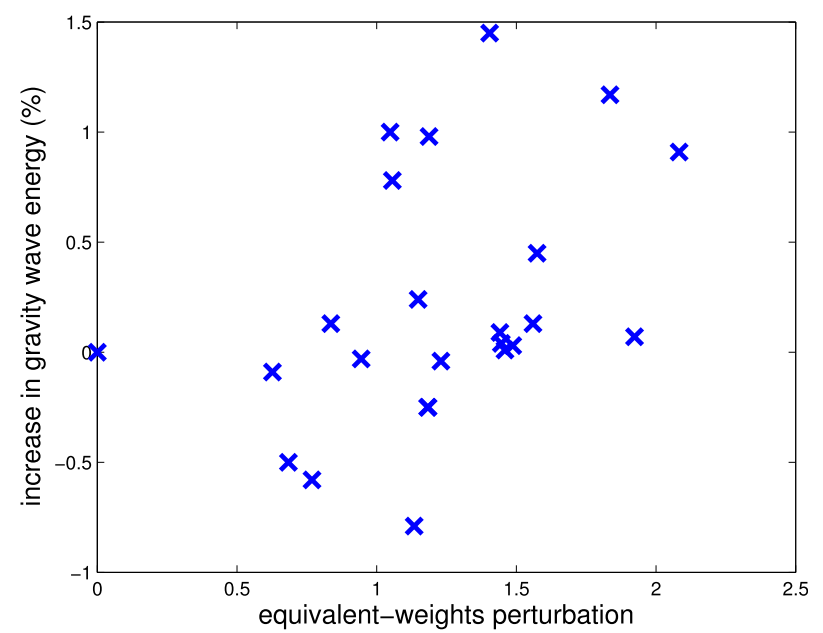

FIG. 5. The size of the $l^{2}$ norm of the equivalent-weights term for each particle plotted against the percentage change in gravity wave energy for the same particle. No relationship between the two is evident.

deterministic model state can be observed by considering the initial condition perturbation (154.8-170.0). Again, no relationship was seen between the size of each of the individual perturbations and the corresponding changes to gravity wave energy (result not shown). However, it does lead to a maximum percentage increase in gravity wave energy $(63174 \%)$, substantially larger than that observed due to the equivalent-weights term $(1.45 \%)$. The initial condition perturbation is based on a sample of random error and so is different from the change in model state due to the equivalentweights term, which is based on $\left[\mathbf{y}^{n}-\mathbf{H} f\left(\mathbf{x}_{i}^{n-1}\right)\right]$ (although both are in geostrophic balance). It does, however, give an indication of the effect of a much larger perturbation and implies that a substantially larger equivalent-weights term may still have the potential to disrupt dynamical balance. Therefore, the issue of the introduction of spurious gravity waves should not be dismissed entirely.

\section{Conclusions}

The majority of data assimilation schemes currently in operational use have a mechanism through which predominantly balanced initial conditions are ensured. The issue with these schemes is that they assume the posterior PDF is a Gaussian, either globally or locally. As the resolution and complexity of numerical models increase, it becomes progressively more important that the full posterior is understood and represented through the initial conditions of forecasts, leading to a better understanding of the future uncertainty. The equivalentweights particle filter is a data assimilation scheme with the potential to represent the full posterior PDF. It avoids the filter degeneracy issue of particle filters through additional terms added to the deterministic model equations. These adaptations again bring into question the problem of ensuring predominantly balanced initial conditions.

This article addresses the potential impact of the equivalent-weights particle filter on dynamical balance and gravity waves through the use of a primitive equation model. The primitive equation model was used since it incorporates both hydrostatic and geostrophic balance, and gravity waves can be present under the parameter settings chosen. To assess the effect of the additional terms required by the equivalent-weights particle filter, the energy spectrum of each particle was calculated. The integral over the energy spectrum associated with the gravity wave dispersion relationship was then evaluated both before and after the addition of the extra terms.

The theoretical examination of the equivalentweights particle filter, together with the gravity wave experiments, lead to four main conclusions: (i) The equivalent-weights particle filter does not cause sufficient imbalance to result in an ensemble mean unrepresentative of the truth. (ii) Provided the model error covariance matrix ensures a geostrophically balanced state through control variable transforms, then each additional term required by the equivalent-weights particle filter is itself in geostrophic balance. (iii) The term associated with the relaxation proposal density (6) actually causes a slight damping in the gravity wave energy if the relaxation term is large enough. (iv) The equivalent-weights term (7) only causes a small increase in gravity wave energy on average, despite leading to a change in model state up to 100 times larger than that induced by the deterministic model equations. The increase can be deemed insignificant when compared to the difference in gravity wave energy due to the permutation of the initial condition. It should be reiterated at this point that variational and EnKF-based methods also produce similar-sized changes in model state in the analysis step.

In summary, the equivalent-weights particle filter is not causing issues related to spurious gravity waves disrupting dynamical balances in this primitive equation model. Although these conclusions imply that the equivalent-weights particle filter is not causing problems, the results are dependent on the model used and the parameters chosen. There is no strong evidence that using a different model or running the model for a longer period of time is likely to change this conclusion. The only caveat to this statement is that some models, such as convection-permitting models, are much more sensitive 
to the initial conditions used. Current data assimilation schemes, both variational methods and the ensemble Kalman filter, are already known to have issues when used in conjunction with such models (Dance 2004; Sun 2005). It may be that the equivalent-weights particle filter will also produce problems in these models. Data assimilation in such sensitive conditions is an area of active research and is one of the many reasons that nonlinear data assimilation methods, such as the equivalent-weights particle filter, are such an important subject to explore.

Acknowledgments. We are grateful to the referees for their clarifying suggestions that have improved the presentation of this material. We would also like to thank the National Centre for Earth Observation (NCEO), part of the National Environment Research Council (NERC), project code H5043718, and the European Space Agency (ESA) project ESA Advanced Data Assimilation Methods, Contract Number ESRIN 4000105001/11/ I-LG, for support via grants.

\section{APPENDIX}

\section{Equivalent-Weights Particle Filter}

The full equivalent-weights particle filter scheme is summarized as follows:

(i) The prior PDF $p\left(\mathbf{x}^{n-r}\right)$ is represented by $N$ individual model states or particles.

(ii) Each particle is run forward to time $n-1$ (the time step immediately preceding the next available observation vector), using the relaxation transition density. If it is assumed that the stochastic term is additive and Gaussian at each time step, this relates to - establishing the new model states using

$$
\mathbf{x}_{i}^{j}=f\left(\mathbf{x}_{i}^{j-1}\right)+\mathbf{B}(\tau)\left[\mathbf{y}^{n}-\mathbf{h}\left(\mathbf{x}_{i}^{j-1}\right)\right]+\widehat{d \boldsymbol{\beta}}_{i}^{j},
$$

where $\widehat{d \boldsymbol{\beta}}_{i}^{j} \sim N(0, \hat{\mathbf{Q}})$; and
- calculating the corresponding weights associated with time step $j$ according to

$$
\begin{aligned}
w_{i}^{j} \propto & \exp \left\{-\frac{1}{2}\left[\mathbf{x}_{i}^{j}-f\left(\mathbf{x}_{i}^{j-1}\right)\right]^{\mathrm{T}} \mathbf{Q}^{-1}\left[\mathbf{x}_{i}^{j}-f\left(\mathbf{x}_{i}^{j-1}\right)\right]\right. \\
& \left.+\frac{1}{2} d \boldsymbol{\beta}_{i}^{\mathrm{T}} \hat{\mathbf{Q}}^{-1} d \boldsymbol{\beta}_{i}\right\}
\end{aligned}
$$

where the product of these weights up to time step $n-1$ is stored for use as $w_{i}^{\text {rest }}$ at the new observation time:

$$
w_{i}^{\mathrm{rest}}=\prod_{j=1}^{n-1} w_{i}^{j} .
$$

(iii) The particles are moved forward to the new observation time $n$ in the last time step according to the equivalent-weights proposal density. The actual steps to calculate the model state for each particle at time $n$ is as follows:

- Calculate the maximum weight it is possible for each particle to achieve via

$$
\begin{aligned}
w_{i}^{\max }= & w_{i}^{\text {rest }} \exp \left[-\frac{1}{2}\left[\mathbf{y}^{n}-\mathbf{H} f\left(\mathbf{x}_{i}^{n-1}\right)\right]^{\mathrm{T}}\right. \\
& \left.\times\left(\mathbf{H Q H} \mathbf{H}^{\mathrm{T}}+\mathbf{R}\right)^{-1}\left[\mathbf{y}^{n}-\mathbf{H} f\left(\mathbf{x}_{i}^{n-1}\right)\right]\right] .
\end{aligned}
$$

- Order the maximum weights for all the particles and then chose $w^{\text {target }}$ such that a certain percentage of particles can achieve it.

- For the chosen percentage of particles where $w_{i}^{\max } \geq w^{\text {target }}$, select the deterministic model state according to

$$
\mathbf{x}_{i}^{*}=f\left(\mathbf{x}_{i}^{n-1}\right)+\alpha_{i} \mathbf{K}\left[\mathbf{y}^{n}-\mathbf{H} f\left(\mathbf{x}_{i}^{n-1}\right)\right],
$$

where $\alpha_{i}$ is defined according to

$$
\begin{aligned}
& \alpha_{i}=1+\sqrt{1-b_{i} / a_{i}}, \\
& \text { where } \quad a_{i}=0.5\left[\mathbf{y}^{n}-\mathbf{H} f\left(\mathbf{x}_{i}^{n-1}\right)\right]^{\mathrm{T}} \mathbf{R}^{-1} \mathbf{H K}\left[\mathbf{y}^{n}-\mathbf{H} f\left(\mathbf{x}_{i}^{n-1}\right)\right] \text { and } \\
& b_{i}=0.5\left[\mathbf{y}^{n}-\mathbf{H} f\left(\mathbf{x}_{i}^{n-1}\right)\right]^{\mathrm{T}} \mathbf{R}^{-1}\left[\mathbf{y}^{n}-\mathbf{H} f\left(\mathbf{x}_{i}^{n-1}\right)\right]+\log w^{\text {target }}-\log w_{i}^{\text {rest }},
\end{aligned}
$$

and $\mathbf{K}=\mathbf{Q} \mathbf{H}^{\mathrm{T}}\left(\mathbf{H Q H}^{\mathrm{T}}+\mathbf{R}\right)^{-1}$. It should be noted that although $\mathbf{K}$ is similar to the Kalman gain matrix, in this case the model error covariance $\mathbf{Q}$ replaces the more standard $\mathbf{P}^{f}$ of the Kalman, or the ensemble Kalman, filter. Otherwise the model state is given by

$$
\mathbf{x}_{i}^{*}=f\left(\mathbf{x}_{i}^{n-1}\right)
$$

- Add stochastic error $\mathbf{Q}^{1 / 2} \boldsymbol{\xi}_{i}^{n}$ to the deterministic model state $\mathbf{x}_{i}^{*}$ to find the final particle model 
state $\mathbf{x}_{i}^{n}$ at time $n$. This error comes from the mixture density given by

$\hat{q}\left(\boldsymbol{\xi}^{n} \mid \mathbf{x}_{i}^{*}\right)=(1-\epsilon) \tilde{\mathbf{U}}_{k}\left(0, \gamma_{\mathbf{U}}\right)+\epsilon N\left(0, \gamma_{N}^{2} \mathbf{l}\right)$.

To sample from the mixture density, a value $u$ is sampled from $u \sim \mathbf{U}[0,1]$. If $u<\epsilon$, then $\boldsymbol{\xi}_{i}^{n} \sim N\left(0, \gamma_{N}^{2} \mathbf{I}\right)$, otherwise $\boldsymbol{\xi}_{i}^{n} \sim \tilde{\mathbf{U}}_{k}\left(0, \gamma_{\mathbf{U}}\right)$.

- The final weight of each particle is then calculated according to

$$
w_{i}^{n}=\frac{w_{i}^{\text {rest }} p\left(\mathbf{x}_{i}^{n} \mid \mathbf{x}_{i}^{n-1}\right) p\left(\mathbf{y}^{n} \mid \mathbf{x}_{i}^{n-1}\right)}{q\left(\mathbf{x}_{i}^{n} \mid \mathbf{x}_{i}^{n-1}, \mathbf{y}^{n}\right)},
$$

where

$$
\begin{aligned}
& w_{i}^{\text {rest }} p\left(\mathbf{x}_{i}^{n} \mid \mathbf{x}_{i}^{n-1}\right) p\left(\mathbf{y}^{n} \mid \mathbf{x}_{i}^{n-1}\right) \\
& =w_{i}^{\text {rest }} \exp \left\{\begin{array}{l}
-\frac{1}{2}\left[\mathbf{x}_{i}^{n}-f\left(\mathbf{x}_{i}^{n-1}\right)\right]^{\mathrm{T}} \mathbf{Q}^{-1}\left[\mathbf{x}_{i}^{n}-f\left(\mathbf{x}_{i}^{n-1}\right)\right] \\
-\frac{1}{2}\left(\mathbf{y}^{n}-\mathbf{H} \mathbf{x}_{i}^{n}\right)^{\mathrm{T}} \mathbf{R}^{-1}\left(\mathbf{y}^{n}-\mathbf{H} \mathbf{x}_{i}^{n}\right)
\end{array}\right\},
\end{aligned}
$$

and

$$
\begin{gathered}
\frac{1}{q\left(\mathbf{x}_{i}^{n} \mid \mathbf{x}_{i}^{n-1}, \mathbf{y}^{n}\right)}=\left\{1+\frac{\epsilon}{(1-\epsilon)}\left(\frac{2}{\pi}\right)^{k / 2}\right. \\
\left.\times \frac{\gamma_{\mathbf{U}}^{k}}{\gamma_{N}} \exp \left[-\frac{1}{2} \boldsymbol{\xi}_{i}^{n^{\mathrm{T}}}\left(\gamma_{N}^{2} \mathbf{l}\right)^{-1} \boldsymbol{\xi}_{i}^{n}\right]\right\}^{-1}, \\
\text { if }-\gamma_{\mathbf{U}} \leq \boldsymbol{\xi}_{i, j} \leq \gamma_{\mathbf{U}}, \forall j=1, \ldots, k, \text { and } \\
\frac{1}{q\left(\mathbf{x}_{i}^{n} \mid \mathbf{x}_{i}^{n-1}, \mathbf{y}^{n}\right)}=\left\{\frac{\epsilon}{(1-\epsilon)}\left(\frac{2}{\pi}\right)^{k / 2}\right. \\
\left.\times \frac{\gamma_{\mathbf{U}}^{k}}{\gamma_{N}} \exp \left[-\frac{1}{2} \boldsymbol{\xi}_{i}^{n^{\mathrm{T}}}\left(\gamma_{N}^{2} \mathbf{l}\right)^{-1} \boldsymbol{\xi}_{i}^{n}\right]\right\}^{-1},
\end{gathered}
$$

otherwise.

(iv) The weights of the particles are normalized by the sum of all the weights of the particles. The ensemble of particles together with their weights now represent the posterior PDF $p\left(\mathbf{x}^{n} \mid \mathbf{y}^{n}\right)$.

(v) Finally, the particles are resampled so that they once again all have weight equal to $1 / N$. This step is required since only the percentage of particles that are able to achieve the target weight will have almost equal weights under the equivalentweights step. The remaining particles will have smaller weights and so will be resampled as duplicate copies of the equivalently weighted particles. The resampled ensemble of particles can now be run forward to the time of the next observation vector.

\section{REFERENCES}

Adcroft, A. J., C. N. Hill, and J. C. Marshall, 1999: A new treatment of the Coriolis terms in C-grid models at both high and low resolutions. Mon. Wea. Rev., 127, 1928-1936, doi:10.1175/ 1520-0493(1999)127<1928:ANTOTC > 2.0.CO;2.

Ades, M., and P. J. Van Leeuwen, 2013: An exploration of the equivalent weights particle filter. Quart. J. Roy. Meteor. Soc., 139, 820-840, doi:10.1002/qj.1995.

— , and — 2014: The equivalent-weights particle filter in a high-dimensional system. Quart. J. Roy. Meteor. Soc., doi:10.1002/qj.2370, in press.

Anderson, J. L., 2001: An ensemble adjustment Kalman filter for data assimilation. Mon. Wea. Rev., 129, 2884-2903, doi:10.1175/1520-0493(2001)129<2884:AEAKFF>2.0.CO;2.

Bannister, R. N., 2008: A review of forecast error covariance statistics in atmospheric variational data assimilation. I: Characteristics and measurements of forecast error covariances. Quart. J. Roy. Meteor. Soc., 134, 1951-1970, doi:10.1002/ qj.339.

Bishop, C. H., B. J. Etherton, and S. J. Majumdar, 2001: Adaptive sampling with the ensemble transform Kalman filter. Part I: Theoretical aspects. Mon. Wea. Rev., 129, 420-436, doi:10.1175/ 1520-0493(2001)129<0420:ASWTET>2.0.CO;2.

Brodeau, L., B. Barnier, A. M. Treguier, T. Penduff, and S. Gulev, 2010: An ERA40-based atmospheric forcing for global ocean circulation models. Ocean Modell., 31, 88-104, doi:10.1016/ j.ocemod.2009.10.005.

Buehner, M., P. Houtekamer, C. Charette, H. L. Mitchell, and B. $\mathrm{He}, 2010$ : Intercomparison of variational data assimilation and the ensemble Kalman filter for global deterministic NWP. Part I: Description and single-observation experiments. Mon. Wea. Rev., 138, 1550-1566, doi:10.1175/ 2009MWR3157.1.

Burgers, G., P. J. van Leeuwen, and G. Evensen, 1998: Analysis scheme in the ensemble Kalman filter. Mon. Wea. Rev., 126, 1719-1724, doi:10.1175/1520-0493(1998)126<1719: ASITEK $>2.0 . \mathrm{CO} ; 2$.

Courtier, P., and O. Talagrand, 1987: Variational assimilation of meteorological observations with the adjoint vorticity equation. II: Numerical results. Quart. J. Roy. Meteor. Soc., 113, 1329-1347, doi:10.1002/qj.49711347813.

Daley, R., 1991: Atmospheric Data Analysis. Cambridge University Press, $457 \mathrm{pp}$.

Dance, S. L., 2004: Issues in high resolution limited area data assimilation for quantitative precipitation forecasting. Physica $D$, 196, 1-27, doi:10.1016/j.physd.2004.05.001.

Doucet, A., N. de Freitas, and N. Gordon, Eds., 2001: Sequential Monte Carlo Methods in Practice. Springer, 581 pp.

Evensen, G., 1994: Sequential data assimilation with a nonlinear quasi-geostrophic model using Monte Carlo methods to forecast error statistics. J. Geophys. Res., 99, 10143-10162, doi:10.1029/94JC00572.

_ 2003: The ensemble Kalman filter: Theoretical formulation and practical implementation. Ocean Dyn., 53, 343-367, doi:10.1007/s10236-003-0036-9.

Gill, A. E., 1982: Atmosphere-Ocean Dynamics. Academic Press, $662 \mathrm{pp}$. 
Houtekamer, P. L., and H. L. Mitchell, 2005: Ensemble Kalman filtering. Quart. J. Roy. Meteor. Soc., 131, 3269-3289, doi:10.1256/qj.05.135.

Kitagawa, G., 1996: Monte Carlo filter and smoother for nonGaussian nonlinear state space models. J. Comput. Graphical Stat., 10, 253-259. [Available online at www.jstor.org/stable/ 1390750.]

Le Dimet, F.-X., and O. Talagrand, 1986: Variational algorithms for analysis and assimilation of meteorological observations: Theoretical aspects. Tellus, 38A, 97-110, doi:10.1111/ j.1600-0870.1986.tb00459.x.

Liu, C., Q. Xiao, and B. Wang, 2008: An ensemble-based fourdimensional variational data assimilation scheme. Part I: Technical formulation and preliminary test. Mon. Wea. Rev., 136, 3363-3373, doi:10.1175/2008MWR2312.1.

Lorenc, A., 1986: Analysis methods for numerical weather prediction. Quart. J. Roy. Meteor. Soc., 112, 1177-1194, doi:10.1002/qj.49711247414.

_, 2003a: Modelling of error covariances by 4D-Var data assimilation. Quart. J. Roy. Meteor. Soc., 129, 3167-3182, doi:10.1256/qj.02.131.

, 2003b: The potential of the ensemble Kalman filter for NWP-A comparison with 4D-Var. Quart. J. Roy. Meteor. Soc., 129, 3183-3203, doi:10.1256/qj.02.132.

, and Coauthors, 2000: The Met. Office global three-dimensional variational data assimilation scheme. Quart. J. Roy. Meteor. Soc., 126, 2991-3012, doi:10.1002/qj.49712657002.
Morzfeld, M., X. Tu, E. Atkins, and A. J. Chorin, 2012: A random map implementation of implicit particle filters. J. Comput. Phys., 231, 2049-2066, doi:10.1016/j.jcp.2011.11.022.

Papadakis, N., E. Mmin, A. Cuzol, and N. Gengembre, 2010: Data assimilation with the weighted ensemble Kalman filter. Tellus, 62A, 673-697, doi:10.1111/j.1600-0870.2010.00461.x.

Snyder, C., T. Bengtsson, P. Bickel, and J. Anderson, 2008: Obstacles to high-dimensional particle filtering. Mon. Wea. Rev., 136, 4629-4640, doi:10.1175/2008MWR2529.1.

Sun, J., 2005: Convective-scale assimilation of radar data: Progresses and challenges. Quart. J. Roy. Meteor. Soc., 131, 34393463, doi:10.1256/qj.05.149.

Talagrand, O., and P. Courtier, 1987: Variational assimilation of meteorological observations with the adjoint vorticity equation. I. Theory. Quart. J. Roy. Meteor. Soc., 113, 1311-1328, doi:10.1002/qj.49711347812.

Van Leeuwen, P. J., 2009: Particle filtering in geophysical systems. Mon. Wea. Rev., 137, 4089-4114, doi:10.1175/2009MWR2835.1.

_ 2010 : Nonlinear data assimilation in geosciences: An extremely efficient particle filter. Quart. J. Roy. Meteor. Soc., 136, 1991-1999, doi:10.1002/qj.699.

Weare, J., 2009: Particle filtering with path sampling and an application to a bimodal ocean current model. J. Comput. Phys., 228, 4312-4331, doi:10.1016/j.jcp.2009.02.033.

Whitaker, J. S., and T. H. Hamill, 2002: Ensemble data assimilation without perturbed observations. Mon. Wea. Rev., 130,1913-1923, doi:10.1175/1520-0493(2002)130<1913:EDAWPO>2.0.CO;2. 